\title{
Research Article Symmetry Properties of Higher-Order Bernoulli Polynomials
}

\author{
Taekyun Kim, ${ }^{1}$ Kyung-Won Hwang, ${ }^{2}$ and Young-Hee Kim $^{1}$ \\ ${ }^{1}$ Division of General Education-Mathematics, Kwangwoon University, Seoul 139-701, South Korea \\ ${ }^{2}$ Department of General Education, Kookmin University, Seoul 136-702, South Korea
}

Correspondence should be addressed to Taekyun Kim, tkkim@kw.ac.kr and

Kyung-Won Hwang, khwang7@kookmin.ac.kr

Received 11 March 2009; Revised 6 July 2009; Accepted 2 August 2009

Recommended by Patricia J. Y. Wong

We investigate properties of identities and some interesting identities of symmetry for the Bernoulli polynomials of higher order using the multivariate $p$-adic invariant integral on $\mathbb{Z}_{p}$.

Copyright (C) 2009 Taekyun Kim et al. This is an open access article distributed under the Creative Commons Attribution License, which permits unrestricted use, distribution, and reproduction in any medium, provided the original work is properly cited.

\section{Introduction}

Let $p$ be a fixed prime number. Throughout this paper $\mathbb{Z}_{p}, \mathbb{Q}_{p}$, and $\mathbb{C}_{p}$ will, respectively, denote the ring of $p$-adic rational integers, the field of $p$-adic rational numbers, and the completion of algebraic closure of $\mathbb{Q}_{p}$. For $x \in \mathbb{C}_{p}$, we use the notation $[x]_{q}=\left(1-q^{x}\right) /(1-q)$. Let $\operatorname{UD}\left(\mathbb{Z}_{p}\right)$ be the space of uniformly differentiable functions on $\mathbb{Z}_{p}$, and let $v_{p}$ be the normalized exponential valuation of $\mathbb{C}_{p}$ with $|p|_{p}=p^{-v_{p}(p)}=1 / p$. For $q \in \mathbb{C}_{p}$ with $|1-q|_{p}<1$, the $q$-Volkenborn integral on $\mathbb{Z}_{p}$ is defined as

$$
I_{q}(f)=\int_{\mathbb{Z}_{p}} f(x) d \mu_{q}(x)=\lim _{N \rightarrow \infty} \frac{1}{\left[p^{N}\right]_{q}} \sum_{x=0}^{p^{N}-1} f(x) q^{x}, \quad f \in \operatorname{UD}\left(\mathbb{Z}_{p}\right)
$$

(see $[1,2])$. The ordinary $p$-adic invariant integral on $\mathbb{Z}_{p}$ is given by

$$
I_{1}(f)=\lim _{q \rightarrow 1} I_{q}(f)=\int_{\mathbb{Z}_{p}} f(x) d x
$$


$($ see $[1-15])$. Let $f^{\prime}(0)=\left.(d f(x) / d x)\right|_{x=0}$. Then we easily see that

$$
I_{1}\left(f_{1}\right)=I_{1}(f)+f^{\prime}(0), \quad \text { where } f_{1}(x)=f(x+1) .
$$

From (1.3), we can derive

$$
\int_{\mathbb{Z}_{p}} e^{x t} d x=\frac{t}{e^{t}-1}=\sum_{n=0}^{\infty} B_{n} \frac{t^{n}}{n !}
$$

(see $[2,8-10])$, where $B_{n}$ are the $n$th Bernoulli numbers.

By (1.2) and (1.3), we easily see that

$$
\begin{aligned}
\frac{n \int_{\mathbb{Z}_{p}} e^{x t} d x}{\int_{\mathbb{Z}_{p}} e^{n x t} d x} & =\frac{1}{t}\left(\int_{\mathbb{Z}_{p}} e^{(x+n) t} d x-\int_{\mathbb{Z}_{p}} e^{x t} d x\right) \\
& =\sum_{i=0}^{n-1} e^{i t}=\sum_{n=0}^{\infty}\left(\sum_{i=0}^{n-1} i^{k}\right) \frac{t^{k}}{k !}=\sum_{k=0}^{\infty} S_{k}(n-1) \frac{t^{k}}{k !},
\end{aligned}
$$

where $S_{k}(n)=0^{k}+1^{k}+\cdots+n^{k}$ for $k \in \mathbb{Z}_{+}$.

It is known that the Bernoulli polynomials are defined by

$$
\int_{\mathbb{Z}_{p}} e^{(x+y) t} d x=\frac{t}{e^{t}-1} e^{x t}=\sum_{n=0}^{\infty} B_{n}(x) \frac{t^{n}}{n !}
$$

where $B_{n}(x)$ are called the $n$th Bernoulli polynomials. The Bernoulli polynomials of order $k$, denoted $B_{n}^{k}(x)$, are defined as

$$
e^{x t}\left(\frac{t}{e^{t}-1}\right)^{k}=\left(\frac{t}{e^{t}-1}\right) \times \cdots \times\left(\frac{t}{e^{t}-1}\right) e^{x t}=\sum_{n=0}^{\infty} B_{n}^{(k)}(x) \frac{t^{n}}{n !}
$$

(see [3-6]). Then the values of $B_{n}^{(k)}(x)$ at $x=0$ are called the Bernoulli numbers of order $k$. When $k=1$, the polynomials or numbers are called the Bernoulli polynomials or numbers. The purpose of this paper is to investigate some interesting properties of symmetry for the multivariate $p$-adic invariant integral on $\mathbb{Z}_{p}$. From the properties of symmetry for the multivariate $p$-adic invariant integral on $\mathbb{Z}_{p}$, we derive some interesting identities of symmetry for the Bernoulli polynomials of higher order.

\section{Symmetry Properties of Higher-Order Bernoulli Polynomials}

Let $w_{1}, w_{2} \in \mathbb{N}$. Then we define

$$
D^{(m)}\left(w_{1}, w_{2}\right)=\left(\frac{w_{1} t}{e^{w_{1} t}-1}\right)^{m} e^{w_{1} w_{2} t x}\left(e^{w_{1} w_{2} t}-1\right)\left(\frac{w_{2} t}{e^{w_{2} t}-1}\right)^{m} \frac{e^{w_{1} w_{2} y t}}{w_{1} w_{2} t} .
$$


From (2.1), we note that

$$
D^{(m)}\left(w_{1}, w_{2}\right)=\frac{\int_{\mathbb{Z}_{p}^{m}} e^{w_{1}\left(x_{1}+x_{2}+\cdots+x_{m}+w_{2} x\right) t} d x_{1} \cdots d x_{m} \int_{\mathbb{Z}_{p}^{m}} e^{w_{2}\left(x_{1}+x_{2}+\cdots+x_{m}+w_{1} y\right) t} d x_{1} \cdots d x_{m}}{\int_{\mathbb{Z}_{p}} e^{w_{1} w_{2} x t} d x},
$$

where $\int_{\mathbb{Z}_{p}^{m}} f\left(x_{1}, \ldots, x_{m}\right) d x_{1} \cdots d x_{m}=\int_{\mathbb{Z}_{p}} \cdots \int_{\mathbb{Z}_{p}} f\left(x_{1}, \ldots, x_{m}\right) d x_{1} \cdots d x_{m}$.

In (2.1), we note that $D^{(m)}\left(w_{1}, w_{2}\right)$ is symmetric in $w_{1}, w_{2}$. By $(2.1)$, we see that

$$
\begin{aligned}
D^{(m)}\left(w_{1}, w_{2}\right)= & \left(\int_{\mathbb{Z}_{p}^{m}} e^{w_{1}\left(x_{1}+\cdots+x_{m}\right) t} d x_{1} \cdots d x_{m}\right) e^{w_{1} w_{2} x t}\left(\frac{\int_{\mathbb{Z}_{p}} e^{w_{2} x_{m} t} d x_{m}}{\int_{\mathbb{Z}_{p}} e^{w_{1} w_{2} x t} d x}\right) \\
& \times\left(\int_{\mathbb{Z}_{p}^{m-1}} e^{w_{2}\left(x_{1}+\cdots+x_{m-1}\right) t} d x_{1} \cdots d x_{m-1}\right) e^{w_{1} w_{2} y t}
\end{aligned}
$$

It is easy to see that

$$
e^{w_{1} w_{2} x t} \int_{\mathbb{Z}_{p}^{m}} e^{w_{1}\left(x_{1}+\cdots+x_{m}\right) t} d x_{1} \cdots d x_{m}=\left(\frac{w_{1} t}{e^{w_{1} t}-1}\right)^{m} e^{w_{1} w_{2} x t}=\sum_{n=0}^{\infty} B_{n}^{(m)}\left(w_{2} x\right) w_{1}^{n} \frac{t^{n}}{n !} .
$$

From (2.1), (2.3), and the above formula, we can derive

$$
\begin{aligned}
D^{(m)}\left(w_{1}, w_{2}\right) & =\left(\sum_{\ell=0}^{\infty} B_{\ell}^{(m)}\left(w_{2} x\right) w_{1}^{\ell} \frac{t^{\ell}}{\ell !}\right)\left(\sum_{k=0}^{\infty} S_{k}\left(w_{1}-1\right) \frac{w_{2}^{k}}{k !} t^{k}\right)\left(\sum_{i=0}^{\infty} B_{i}^{(m-1)}\left(w_{1} y\right) \frac{w_{2}^{i}}{i !} t^{i}\right) \frac{1}{w_{1}} \\
& =\left(\sum_{\ell=0}^{\infty} B_{\ell}^{(m)}\left(w_{2} x\right) w_{1}^{\ell-1} \frac{t^{\ell}}{\ell !}\right)\left(\sum_{j=0}^{\infty}\left(\sum_{k=0}^{j} S_{k}\left(w_{1}-1\right) w_{2}^{k} w_{2}^{j-k} \frac{B_{j-k}^{(m-1)}\left(w_{1} y\right)}{k !(j-k) !} j !\right) \frac{t^{j}}{j !}\right) \\
& =\sum_{n=0}^{\infty}\left(\sum_{j=0}^{n}\left(\begin{array}{l}
n \\
j
\end{array}\right) w_{2}^{j} w_{1}^{n-j-1} B_{n-j}^{(m)}\left(w_{2} x\right) \sum_{k=0}^{j} S_{k}\left(w_{1}-1\right)\left(\begin{array}{l}
j \\
k
\end{array}\right) B_{j-k}^{(m-1)}\left(w_{1} y\right)\right) \frac{t^{n}}{n !} .
\end{aligned}
$$

By the symmetry of $D^{(m)}\left(w_{1}, w_{2}\right)$ in $w_{1}$ and $w_{2}$, we see that

$$
D^{(m)}\left(w_{1}, w_{2}\right)=\sum_{n=0}^{\infty}\left(\sum_{j=0}^{n}\left(\begin{array}{l}
n \\
j
\end{array}\right) w_{1}^{j} w_{2}^{n-j-1} B_{n-j}^{(m)}\left(w_{1} x\right) \sum_{k=0}^{j}\left(\begin{array}{l}
j \\
k
\end{array}\right) S_{k}\left(w_{2}-1\right) B_{j-k}^{(m-1)}\left(w_{2} y\right)\right) \frac{t^{n}}{n !} .
$$


By comparing the coefficients on both sides of (2.5) and (2.6), we obtain the following theorem.

Theorem 2.1. For $w_{1}, w_{2} \in \mathbb{N}, n \geq 0, m \geq 1$, one has

$$
\begin{aligned}
& \sum_{j=0}^{n}\left(\begin{array}{l}
n \\
j
\end{array}\right) w_{2}^{j} w_{1}^{n-j-1} B_{n-j}^{(m)}\left(w_{2} x\right) \sum_{k=0}^{j} S_{k}\left(w_{1}-1\right)\left(\begin{array}{l}
j \\
k
\end{array}\right) B_{j-k}^{(m-1)}\left(w_{1} y\right) \\
& =\sum_{j=0}^{n}\left(\begin{array}{l}
n \\
j
\end{array}\right) w_{1}^{j} w_{2}^{n-j-1} B_{n-j}^{(m)}\left(w_{1} x\right) \sum_{k=0}^{j}\left(\begin{array}{l}
j \\
k
\end{array}\right) S_{k}\left(w_{2}-1\right) B_{j-k}^{(m-1)}\left(w_{2} y\right) .
\end{aligned}
$$

Let $y=0$ and $m=1$ in (2.7). Then we have the following corollary.

Corollary 2.2. For $n \in \mathbb{Z}_{+}$, one has

$$
\begin{aligned}
& \sum_{j=0}^{n}\left(\begin{array}{l}
n \\
j
\end{array}\right) w_{1}^{n-j-1} w_{2}^{j} B_{n-j}\left(w_{2} x\right) S_{j}\left(w_{1}-1\right) \\
& \quad=\sum_{j=0}^{n}\left(\begin{array}{l}
n \\
j
\end{array}\right) w_{1}^{j} w_{2}^{n-j-1} B_{n-j}\left(w_{1} x\right) S_{j}\left(w_{2}-1\right) .
\end{aligned}
$$

If we take $w_{2}=1 \mathrm{in}(2.8)$, then we also obtain the following corollary.

Corollary 2.3. For $w_{1} \in \mathbb{N}$, one has

$$
B_{n}\left(w_{1} x\right)=\sum_{i=0}^{n}\left(\begin{array}{l}
n \\
i
\end{array}\right) w_{1}^{i-1} B_{i}(x) S_{n-i}\left(w_{1}-1\right)
$$

By the definition of $D^{(m)}\left(w_{1}, w_{2}\right)$, we easily see that

$$
\begin{aligned}
D^{(m)}\left(w_{1}, w_{2}\right) & =\left(\frac{w_{1} t}{e^{w_{1} t}-1}\right)^{m} e^{x w_{1} w_{2} t} \frac{e^{w_{1} w_{2} t}-1}{e^{w_{2} t}-1}\left(\frac{w_{2} t}{e^{w_{2} t}-1}\right)^{m-1} e^{y w_{1} w_{2} t} \frac{1}{w_{1}} \\
& =\frac{1}{w_{1}}\left(\sum_{i=0}^{w_{1}-1} \sum_{k=0}^{\infty} B_{k}^{(m)}\left(w_{2} x+\frac{w_{2}}{w_{1}} i\right) w_{1}^{k} \frac{t^{k}}{k !}\right)\left(\sum_{\ell=0}^{\infty} B_{\ell}^{(m-1)}\left(w_{1} y\right) w_{2}^{\ell} \frac{t^{\ell}}{\ell !}\right) \\
& =\sum_{n=0}^{\infty}\left(\sum_{k=0}^{n}\left(\sum_{i=0}^{w_{1}-1} B_{k}^{(m)}\left(w_{2} x+\frac{w_{2}}{w_{1}} i\right)\right) \frac{w_{1}^{k-1}}{k !} B_{n-k}^{(m-1)}\left(w_{1} y\right) \frac{w_{2}^{n-k}}{(n-k) !} n !\right) \frac{t^{n}}{n !} \\
& =\sum_{n=0}^{\infty}\left(\sum_{k=0}^{n}\left(\begin{array}{l}
n \\
k
\end{array}\right) w_{1}^{k-1} w_{2}^{n-k} B_{n-k}^{(m-1)}\left(w_{1} y\right) \sum_{i=0}^{w_{1}-1} B_{k}^{(m)}\left(w_{2} x+\frac{w_{2}}{w_{1}} i\right)\right) \frac{t^{n}}{n !} .
\end{aligned}
$$


From the symmetric property of $D^{(m)}\left(w_{1}, w_{2}\right)$ in $w_{1}, w_{2}$, we note that

$$
D^{(m)}\left(w_{1}, w_{2}\right)=\sum_{n=0}^{\infty}\left(\sum_{k=0}^{n}\left(\begin{array}{l}
n \\
k
\end{array}\right) w_{2}^{k-1} w_{1}^{n-k} B_{n-k}^{(m-1)}\left(w_{2} y\right) \sum_{i=0}^{w_{2}-1} B_{k}^{(m)}\left(w_{1} x+\frac{w_{1}}{w_{2}} i\right)\right) \frac{t^{n}}{n !} .
$$

By comparing the coefficients on both sides of (2.10) and (2.11), we obtain the following theorem.

Theorem 2.4. For $w_{1}, w_{2} \in \mathbb{N}, n \in \mathbb{Z}_{+}, m \in \mathbb{N}$, one has

$$
\begin{aligned}
\sum_{k=0}^{n}\left(\begin{array}{l}
n \\
k
\end{array}\right) w_{1}^{k-1} w_{2}^{n-k} B_{n-k}^{(m-1)}\left(w_{1} y\right) \sum_{i=0}^{w_{1}-1} B_{k}^{(m)}\left(w_{2} x+\frac{w_{2}}{w_{1}} i\right) \\
=\sum_{k=0}^{n}\left(\begin{array}{l}
n \\
k
\end{array}\right) w_{2}^{k-1} w_{1}^{n-k} B_{n-k}^{(m-1)}\left(w_{2} y\right) \sum_{i=0}^{w_{2}-1} B_{k}^{(m)}\left(w_{1} x+\frac{w_{1}}{w_{2}} i\right) .
\end{aligned}
$$

Let $y=0$ and $m=1$ in (2.12). Then we obtain the following Corollary 2.5 .

Corollary 2.5. For $w_{1}, w_{2} \in \mathbb{N}$, one has

$$
w_{1}^{n-1} \sum_{i=0}^{w_{1}-1} B_{n}\left(w_{2} x+\frac{w_{2}}{w_{1}} i\right)=w_{2}^{n-1} \sum_{i=0}^{w_{2}-1} B_{n}\left(w_{1} x+\frac{w_{1}}{w_{2}} i\right)
$$

From (2.12), we can get the well-known result due to Raabe:

$$
\sum_{i=0}^{w_{1}-1} B_{n}\left(x+\frac{1}{w_{1}} i\right)=w_{1}^{1-n} B_{n}\left(w_{1} x\right)
$$

\section{References}

[1] T. Kim, "Symmetry p-adic invariant integral on $\mathbb{Z}_{p}$ for Bernoulli and Euler polynomials," Journal of Difference Equations and Applications, vol. 14, no. 12, pp. 1267-1277, 2008.

[2] T. Kim, "On a $q$-analogue of the $p$-adic log gamma functions and related integrals," Journal of Number Theory, vol. 76, no. 2, pp. 320-329, 1999.

[3] M. Abramowitz and I. A. Stegun, Handbook of Mathematical Functions, National Bureau of Standards, 1964.

[4] Ch. Jordan, Calculus of Finite Differences, Chelsea, New York, NY, USA, 2nd edition, 1950.

[5] L. M. Milne-Thomson, The Calculus of Finite Differences, Macmillan, London, UK, 1933.

[6] N. E. Nörlund, Vorlesungen über Differenzenrechnung, Springer, Berlin, Germany, 1924.

[7] Y. H. Kim, "On the $p$-adic interpolation functions of the generalized twisted $(h, q)$-Euler numbers," International Journal of Mathematical Analysis, vol. 3, pp. 897-904, 2009.

[8] T. Kim, " $q$-Volkenborn integration," Russian Journal of Mathematical Physics, vol. 9, no. 3, pp. 288-299, 2002.

[9] T. Kim, " $q$-Bernoulli numbers and polynomials associated with Gaussian binomial coefficients," Russian Journal of Mathematical Physics, vol. 15, no. 1, pp. 51-57, 2008.

[10] T. Kim, "Analytic continuation of multiple q-zeta functions and their values at negative integers," Russian Journal of Mathematical Physics, vol. 11, no. 1, pp. 71-76, 2004. 
[11] T. Kim, "Non-Archimedean $q$-integrals associated with multiple Changhee $q$-Bernoulli polynomials," Russian Journal of Mathematical Physics, vol. 10, no. 1, pp. 91-98, 2003.

[12] T. Kim, "Symmetry of power sum polynomials and multivariate fermionic $p$-adic invariant integral on $\mathbb{Z}_{p}$, " Russian Journal of Mathematical Physics, vol. 16, no. 1, pp. 93-96, 2009.

[13] H. Ozden and Y. Simsek, "A new extension of $q$-Euler numbers and polynomials related to their interpolation functions," Applied Mathematics Letters, vol. 21, no. 9, pp. 934-939, 2008.

[14] Y. Simsek, "On $p$-adic twisted $q$ - $L$-functions related to generalized twisted Bernoulli numbers," Russian Journal of Mathematical Physics, vol. 13, no. 3, pp. 340-348, 2006.

[15] Y.-H. Kim and K.-W. Hwang, "A symmetry of power sum and twisted Bernoulli polynomials," Advanced Studies in Contemporary Mathematics, vol. 18, no. 2, pp. 127-133, 2009. 